\title{
RESULTADOS PRELIMINARES DA DISTRIBUIÇÃO DE FÓSFORO E SUAS FORMAS NOS SEDIMENTOS DA PLATAFORMA CONTINENTAL DO ESTADO DE SANTA CATARINA
}

\author{
PELLENS, I.C.; BELLOTTO, V.R.; KUROSHIMA, K.N. \& J.G.N. ABREU \\ CTTMar - UNIVALI \\ Rua Uruguai, 458, Cx.P. 360, Itajaí, SC - CEP 88.302-202
}

\begin{abstract}
RESUMO
O estudo de fósforo em sedimentos marinhos tem sido utilizado como importante ferramenta para compreensão do ciclo geoquímico deste nutriente, o qual vem sendo alterado pela ação antropogênica. Os sedimentos marinhos representam o depósito final deste constituinte e, dependendo dos processos de interação química que ali ocorrem, podem ser responsáveis por sua maior ou menor disponibilidade para a coluna d'água. Neste estudo foram determinadas as diferentes formas de fósforo, através do método de extração sequencial (modificado de SILVEIRA, 1993), em 27 amostras de sedimentos superficiais coletados na plataforma interna do litoral centro-norte catarinense. O objetivo era verificar a distribuição de fósforo e suas formas de acordo com as fácies sedimentares encontradas na área de estudo. As concentrações de fósforo total variaram entre 1,27 a $18,77 \mu \mathrm{mol} / \mathrm{g}$, com as mais elevadas estando associadas aos sedimentos finos, embora tenham ocorrido diferenças entre fácies com características granolumétricas similares. O fosfato de ferro + alumínio apresentou concentrações mais altas próximo as desembocaduras dos rios diminuindo com o aumento da distância da linha de costa, indicando o aporte continental. A forma predominante na maioria das amostras foi o fosfato de cálcio que apresentou uma relação direta com a profundidade.
\end{abstract}

Palavras chave: fósforo, sedimentos costeiros, extração sequencial.

\section{PRELIMINARY RESULTS ON THE DISTRIBUTION OF PHOSPHORUS AND ITS FORMS IN THE CONTINENTAL SHELF SEDIMENTS OFF SANTA CATARINA STATE, BRAZIL}

\begin{abstract}
Distribuition of phosphorus in marine sediments have been recognized as a useful tool for the knowledgement of its geochemical cicle, that have been undergoing chances by human actions. Marine sediments represents the major reservoir of this constituint, where processes take place and control its disponibility to water column. Differents forms of phosphorus were determined by sequencial extrations in 27 samples of bottom sediment collected in the inner shelf off Santa Catarina. The aim of this study was to investigate the distribuition of phosphorus in the sequencial extrated frations and the relationship with sediment facies. The highest total phosphorus concentrations have been related to fine grained sediments. Although, there were some differences between samples with similar sedimentar patterns. Iron plus aluminium phosphate has been showed high concentrations in the neigborhood of river mounths, declining off shore. Calcium phosphate was the predominant form in most samples.
\end{abstract}

Key words: phosphorus, coastal sediments, sequential extraction. 
PELLENS et al.: Fosforo e suas formas em sedimentos de Santa Catarina.

\section{INTRODUÇÃO}

O fósforo existente nos oceanos é principalmente de origem continental e, chega ao fundo marinho conduzido pelos rios, após sofrer diversos processos intempéricos, químicos e físicos.

A taxa de suprimento deste constituinte para os oceanos depende, em última instância, do clima e do tipo de rochas continentais, embora condições físicas como direção de ventos, correntes e topografia também devam ser consideradas (Barron \& Frakes, 1990; Boström et al., 1988).

Por outro lado, o fósforo é um dos nutrientes utilizados para a formação de biomassa nos sistemas marinhos e é reciclado no próprio meio (Dolan, 1997). Parte deste constituinte passa pelo ciclo orgânico e acaba sendo imobilizado nos sedimentos, principalmente como fosfato de cálcio ou de ferro.

A distribuição das diferentes formas de fósforo pode estar relacionada aos diferentes tipos de sedimentos, estando associada aos processos de interação química deste elemento com as partículas orgânicas e inorgânicas depositadas. Desta forma, os sedimentos atuam como reservatórios em sistemas naturais e podem ter importante função na retenção de fósforo, através de processos de adsorção, precipitação autigênica e assimilação biológica (Edmond et al., 1981; Fox, 1989; Lebo, 1991, in Vink et al., 1997) e, na sua liberação para a coluna d'água por processos de desorção, dissolução e decomposição de matéria orgânica (Berner, 1990). Estas reações ocorrem tanto em depósitos sedimentares, quanto na coluna d'água, tornando seus efeitos relativos no transporte de fósforo para o oceano, difíceis de determinar.

Além disso, estudos tem demonstrado que o Homem está interferindo no ciclo do fósforo através de ações como desmatamentos, mau uso dos solos (Odum, 1983) e lançamento de efluentes urbanos, industriais e agrícolas que são transportados para o ambiente marinho. Isso contribui para um aumento deste constituinte nos oceanos, desequilibrando o seu ciclo geoquímico.

Diferenças entre os padrões de deposição de fósforo em corpos aquosos são refletidas pela sua composição nos sedimentos. A composição sedimentar fracionada é normalmente obtida pela utilização de métodos de extrações químicas seqüenciais (Silveira, 1993) e as frações são normalmente definidas operacionalmente (Logan, 1982; Peterson et al., 1988), embora isso não signifique que as mesmas correspondam a mecanismos específicos de retenção ou ligação a compostos sedimentares (Bostrom et al., 1988).

O objetivo deste trabalho foi determinar a distribuição das diferentes formas de fósforo nas distintas fácies sedimentares da plataforma continental interna catarinense. Além disso, por ser a primeira investigação realizada nesta área, poderá servir de subsídio para pesquisas futuras visando avaliar a influência antropogênica no ciclo geoquímico deste elemento e suas implicações para o ambiente em questão.

\section{ÁREA DE ESTUDO}

A área estudada abrange a plataforma interna do litoral centro-norte catarinense, compreendida entre os rios Itapocu e Tijucas, en-

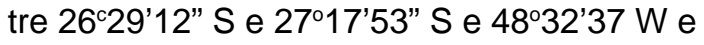
$48^{\circ} 34^{\prime} 23^{\prime \prime W}$ (Fig.1), sendo o limite leste a isóbata de 50 metros.

Nesta área desembocam três rios, com características distintas entre si:

1. Rio Itapocu: desemboca em um sistema ilha-barreira;

2. Rio Itajaí-Açu: desembocando diretamente em mar aberto;

3. Rio Tijucas: que desemboca em uma baía.

\section{MATERIAL E MÉTODOS}

A coleta de amostras de sedimento foi realizada durante um cruzeiro oceanográfico, 


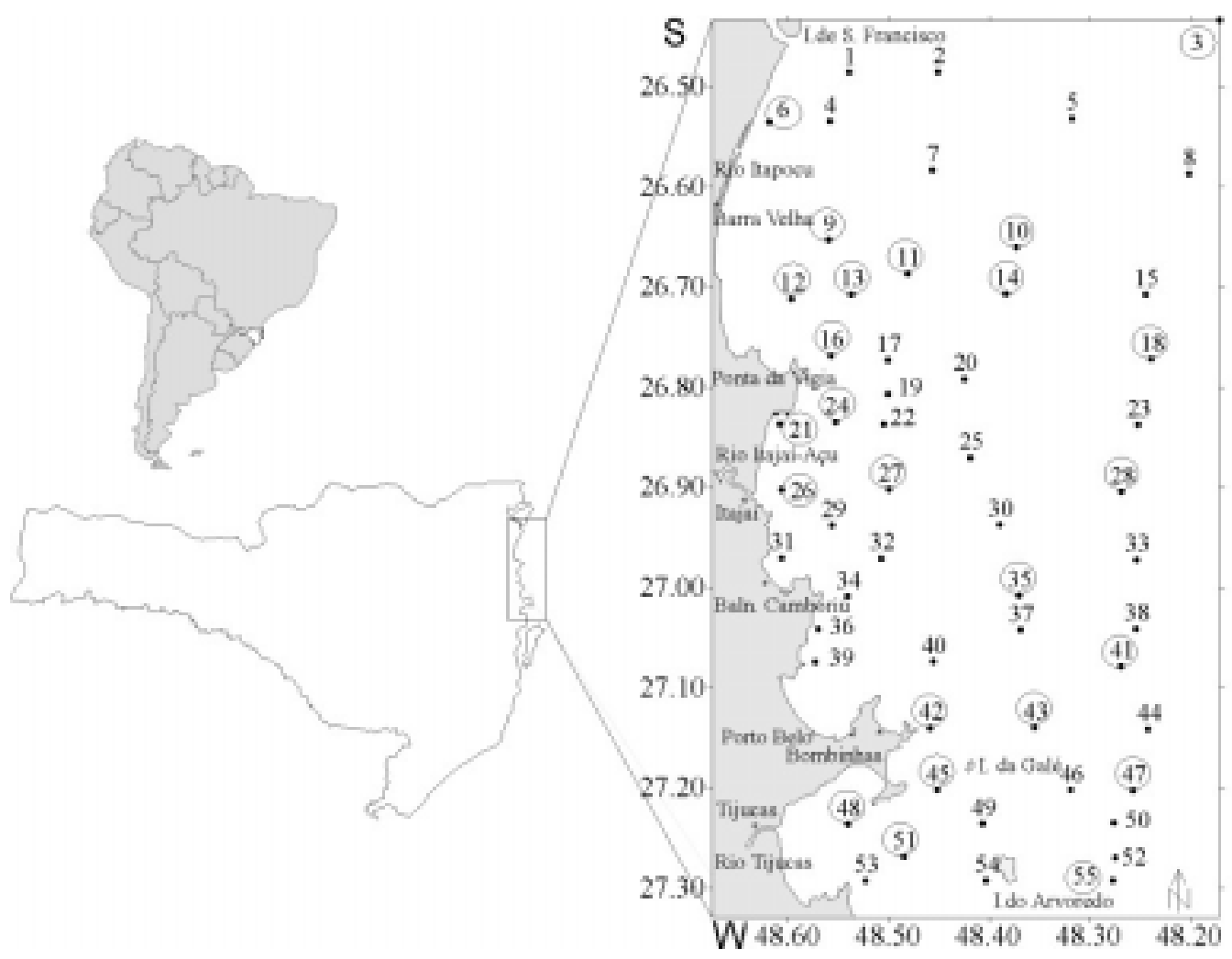

Figura 1 - Mapa da região de estudo localizando as estações de coleta de sedimentos e, circuladas, as amostras selecionadas para a determinação de fósforo e suas formas.

entre os dias 27 de fevereiro a 02 de março de 1996, a bordo do navio de pesquisa N/Pq Diadorim (CEPSULIIBAMA). Os sedimentos foram coletados em 55 pontos amostrais distribuídos em 21 perfis transversais à linha de costa (Fig.1), com draga de fundo tipo Van Veen. De cada amostra foram retirados, com auxílio de uma seringa $(2 \mathrm{~cm}$ de diâmetro e $7 \mathrm{~cm}$ de altura), os primeiros $10 \mathrm{~cm}$ superficiais do sedimento para determinação de fósforo. Estas subamostras foram acondicionadas em sacos plásticos etiquetados e imediatamente levadas ao freezer, onde permaneceram até o momento das análises. O restante do material coletado foi reservado para as análises sedimentológicas.
Após a análise granulométrica dos sedimentos, foi feita uma seleção das amostras, de modo a abranger todas as fácies compreendidas na área de estudo, nas quais, foram determinados os conteúdos de matéria orgânica e carbonato.

As amostras reservadas para determinação de fósforo e suas formas foram descongeladas, secas em estufa a aproximadamente $50^{\circ} \mathrm{C}$ e, depois de resfriadas, foram reduzidas a pó fino, utilizando gral com pistilo. De cada amostra foram pesadas alíquotas de 0,1 grama (em triplicata), as quais seguiram as etapas extração seqüencial de fósforo segundo a figura 2, modificado a partir de Silveira (1993). 


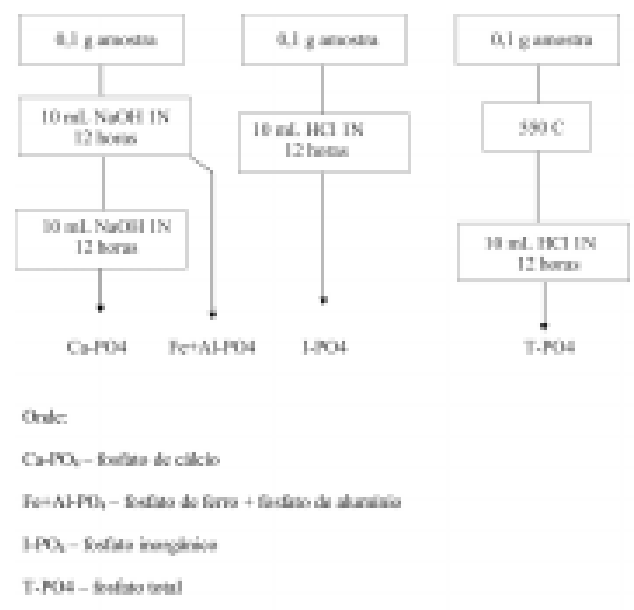

Figura 2 - Esquema de extração sequencial de fósforo (modificado de SILVEIRA, 1993).

Estas subamostras destinadas à determinação de $\mathrm{Al}-\mathrm{PO}_{4}+\mathrm{Fe}-\mathrm{PO}_{4}, \mathrm{Ca}-\mathrm{PO}_{4}, \mathrm{O}-\mathrm{PO}_{4}$ e $\mathrm{I}_{-} \mathrm{PO}_{4}$ foram colocadas em tubos de polipropileno cônicos com tampa e capacidade volumétrica de $15 \mathrm{~mL}$. A cada tubo foram adicionados $10 \mathrm{~mL}$ de reagente (específico para cada fase de extração; segundo o esquema). Os tubos fechados foram levados à mesa agitadora, onde permaneceram por 12 horas sob uma velocidade de $70 \mathrm{rpm}$. Após este período de agitação, as amostras foram centrifugadas durante 3 minutos, a aproximadamente $2500 \mathrm{rpm}$. Dos extratos obtidos foi retirado $1 \mathrm{~mL}$, diluído em $15 \mathrm{~mL}\left(\mathrm{Al}-\mathrm{PO}_{4}+\right.$ $\mathrm{Fe}-\mathrm{PO}_{4}, \mathrm{Ca}-\mathrm{PO}_{4}$ e I-PO $\mathrm{PO}_{4}$ ) ou $30 \mathrm{~mL}\left(\mathrm{O}-\mathrm{PO}_{4}\right)$ de água destilada e, aos quais, foi adicionada a mistura de reagentes para determinação na forma de fosfato (Strickland \& Parsons, 1972). A concentração de fósforo (como fosfato) foi determinada por espectrofotometria de luz visível (Shimadzu), em comprimento de onda de $885 \mathrm{~nm}$, utilizando-se cubetas de 5 $\mathrm{cm}$ de trajeto óptico.

\section{RESULTADOS E DISCUSSÃO}

Os sedimentos transportados até a região costeira por diferentes agentes perma- necem depositados nas margens continentais onde ficam sujeitos a diferentes condições ambientais, físicas e químicas. Estes sedimentos incluem cascalhos, areias, siltes e argilas, de origem terrígena e biogênica, sendo o último formado principalmente por restos de conchas de moluscos. Os sedimentos de granulometria fina e grosseira são encontrados em ambientes de baixa e alta energia, respectivamente (Chester, 1990).

A distribuição de fósforo e suas formas em sedimentos pode ser melhor interpretada quando relacionada com dados de granulometria. Neste estudo a soma das frações silte e argila foi considerada como sedimentos finos e as frações areia e cascalho foram denominadas simplesmente de sedimentos grosseiros, por não haver necessidade de um maior detalhamento granulométrico.

A distribuição dos sedimentos superficiais da plataforma interna do litoral centronorte de Santa Catarina (Fig.3) mostra que os rios Itajaí-Açu e Tijucas apresentam considerável contribuição de sedimentos finos. $O$ Rio Itapocu, entretanto, apresenta uma maior contribuição com respeito aos sedimentos grosseiros depositados na margem da plataforma.

Os sedimentos finos (Fig. 3) apresentaram os maiores teores de matéria orgânica (Fig. 4) e carbonato (Fig.5) os quais estiveram associados às áreas próximas das desembocaduras dos rios Itajaí-Açu e Tijucas, da região próxima das Ilhas da Galé e Arvoredo ( estações 42 e 45) e, de algumas estações localizadas na isóbata de 50 metros (estações 18, 28, 41, 47 e 55).

Os resultados sobre a distribuição das diferentes formas de fósforo nos sedimentos superficiais da plataforma interna do Estado de Santa Catarina estão apresentados em concentração na Tabela 1 e, em porcentagem na Tabela 2, onde também são mostrados os percentuais de sedimentos finos e grosseiros, de matéria orgânica e de carbonato.

O fósforo total (Fig. 6A) e suas frações (Fig. 6B, 6C, 7A e 7B) estão associados, de 
forma geral, aos sedimentos finos (Fig. 3). As maiores concentrações foram encontradas nas áreas sob influência dos rios Itaja-Açu e Tijucas, com tendência de aumento em direção sul, onde se estendem até a isóbata de $50 \mathrm{~m}$. Exceção a este padrão geral, foi o fosfato de ferro + alumínio (Fig. 7A) que apresentou concentrações mais elevadas restritas às desembocaduras dos rios, decrescendo com o aumento da profundidade. Este padrão pode ser explicado pela maior disponibilidade de $\mathrm{Fe}$ e Al nas regiões próximas à costa, as quais apresentam condições favoráveis para a precipitação destes constituintes. Segundo Rao \& Berner (1997) o fosfato de ferro + alumínio está associado aos grãos finos porque estes possuem maior razão superfície/volume para a adsorção de colóides de ferro. A maior concentração desta fração foi detectada na estação 26, localizada próxima a desembocadura do Rio Itajaí-Açu. Esta localização provavelmente contribuiu para o aumento de fosfato de ferro + alumínio, devido a maior descarga de material em suspensão que, segundo Bellotto et al. (1997), pode chegar a $34 \mathrm{mg} / \mathrm{L}$, favorecendo os processos de adsorção. Em estudo realizado ao longo da plataforma brasileira, Silveira (1993) verificou que as regiões próximas a foz do Rio Amazonas (Brasil) também apresentaram concentrações elevadas desta fração, o que foi atribuído ao aumento do material em suspensão nesta região.

O fósforo inorgânico (Fig. 6B) e o orgânico (Fig. 6C) estiveram mais freqüentemente

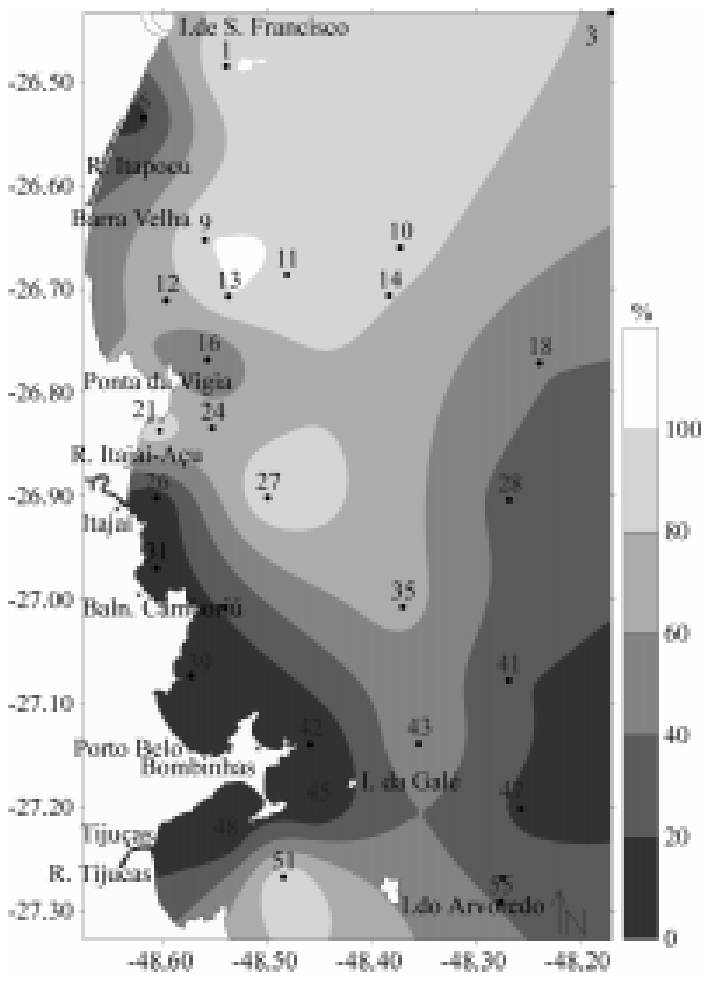

Figura 3 - Distribuição percentual dos sedimentos superficiais na plataforma interna do litoral centro-norte de Santa Catarina.

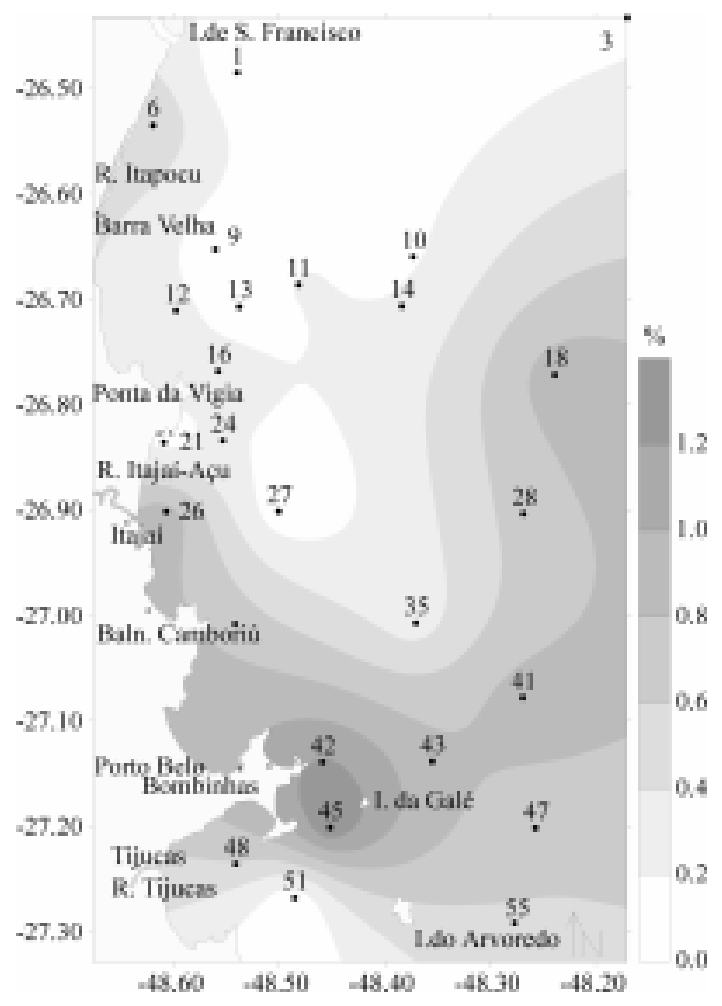

Figura 4 - Distribuição da matéria orgânica (\%) nos sedimentos superficiais da plataforma interna do centronorte de Santa Catarina. 


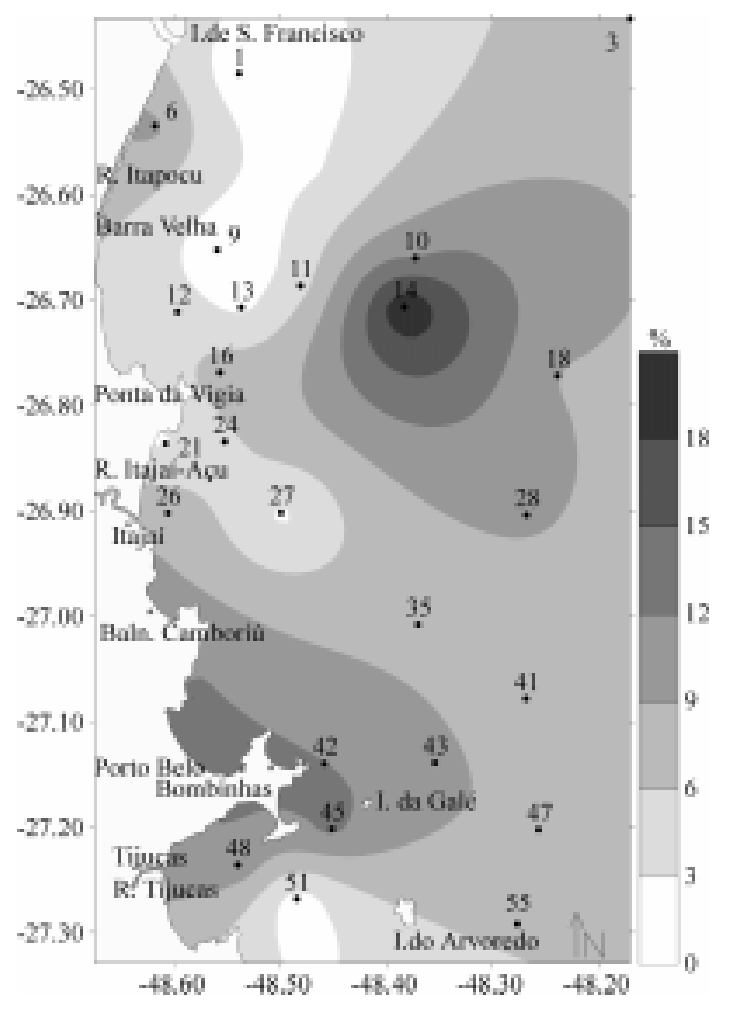

Figura 5 - Distribuição de carbonatos (\%), no sedimento superficial da plataforma interna do centro-norte de Santa Catarina. associados aos sedimentos finos e apresentaram menores concentrações na região norte da área amostrada. Estas duas frações apresentaram padrão de distribuição bastante similar ao do fósforo total. Testes de correlação entre as concentrações de fósforo total versus fósforo inorgânico ( $r=0.92)$ e fósforo orgânico $(r=0.93)$ indicaram existir uma boa relação entre estas formas. Segundo Williams et al. (1971), isto indica que tanto as fontes de fósforo inorgânico quanto as de orgânico são importantes para a deposição de fósforo no sedimento.

O fosfato de cálcio (Fig. 7B) foi a fração predominante entre as formas inorgânicas na maioria das estações, apresentando maiores concentrações próximo a foz dos rios.

\section{Distribuição Percentual}

Tanto o fósforo orgânico, quanto o inorgânico (Fig. 8A e 8B) apresentaram maiores porcentagens na fração mais fina dos sedimentos, notadamente próximo a llha de São Francisco e na região de Porto Belo e Bombinhas. Observa-se que houve uma menor contribuição de aporte continental com

Tabela 1 - Concentrações médias de fósforo total, inorgânico, orgânico, fosfato de ferro + alumínio e fosfato e cálcio (mmol/g) na área de estudo, bem como, mínimos, máximos, e desvio padrão.

\begin{tabular}{|c|c|c|c|c|c|}
\hline Estação & $\begin{array}{c}\mathrm{T}-\mathrm{PO}_{4} \\
(\mu \mathrm{mol} / \mathrm{g})\end{array}$ & $\begin{array}{c}\mathrm{I}^{-\mathrm{PO}_{4}} \\
(\mu \mathrm{mol} / \mathrm{g})\end{array}$ & $\begin{array}{c}\mathrm{O}^{-\mathrm{PO}_{4}} \\
(\mu \mathrm{mol} / \mathrm{g})\end{array}$ & $\begin{array}{c}\mathrm{Fe}+\mathrm{Al}-\mathrm{PO}_{4} \\
(\mu \mathrm{mol} / \mathrm{g})\end{array}$ & $\begin{array}{c}\mathrm{Ca}-\mathrm{PO}_{4} \\
(\mu \mathrm{mol} / \mathrm{g})\end{array}$ \\
\hline Máximo & 18,77 & 13,16 & 7,39 & 6,97 & 7,72 \\
\hline Mínimo & 1,27 & 0,80 & 0,10 & 0,27 & 0,53 \\
\hline Média & 8,56 & 6,24 & 2,32 & 1,72 & 4,52 \\
\hline D.Padrão & 5,01 & 3,47 & 1,71 & 1,46 & 2,37 \\
\hline
\end{tabular}

Tabela 2 - Valores percentuais médios de sedimentos grosseiros e finos, matéria orgânica e carbonato e, do fósforo e suas formas na área de estudo, bem como os ínimos, máximos, e desvio padrão de cada parâmetro.

\begin{tabular}{lcccccccc}
\hline \hline Estação & $\begin{array}{c}\text { Grosseiros } \\
(\%)\end{array}$ & $\begin{array}{c}\text { Finos } \\
(\%)\end{array}$ & $\begin{array}{c}\mathrm{I}^{-\mathrm{PO}_{4}} \\
(\%)\end{array}$ & $\begin{array}{c}\mathrm{O}^{-\mathrm{PO}_{4}} \\
(\%)\end{array}$ & $\begin{array}{c}\mathrm{Fe}+\mathrm{Al}-\mathrm{PO}_{4} \\
(\%)\end{array}$ & $\begin{array}{c}\mathrm{Ca}^{2} \mathrm{PO}_{4} \\
(\%)\end{array}$ & $\begin{array}{c}\mathrm{MO} \\
(\%)\end{array}$ & $\begin{array}{c}\text { Carbonato } \\
(\%)\end{array}$ \\
\hline Mínimo & 0,0 & 0,1 & 57,3 & 7,6 & 10,8 & 37,1 & 1,37 & 20,86 \\
Máximo & 99,9 & 100,0 & 92,4 & 42,7 & 45,5 & 71,1 & 0,02 & 0,90 \\
Média & 44,6 & 55,4 & 74,7 & 25,3 & 21,5 & 53,8 & 0,49 & 7,49 \\
D.Padrão & 38,2 & 38,2 & 9,2 & 9,2 & 10,3 & 9,5 & 0,40 & 4,53 \\
\hline \hline
\end{tabular}



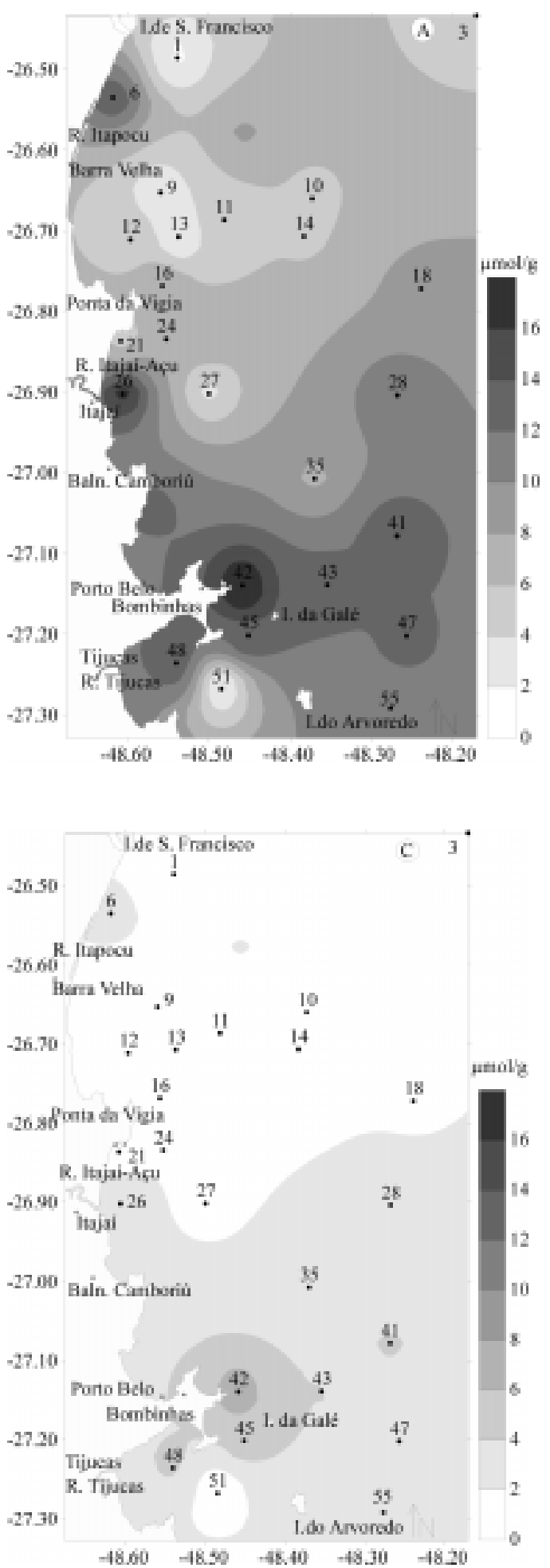

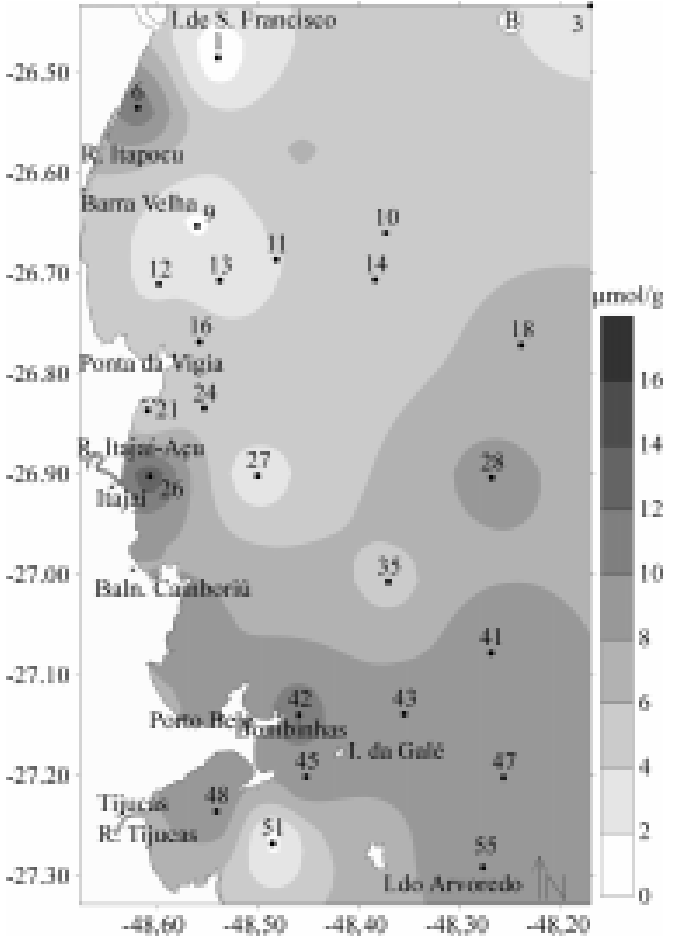

Figura 6 - Distribuição espacial de fósforo total (A), inorgânico (B) e orgânico (C), (mmol/g), nos sedimentos superficiais da plataforma interna do litoral centro-norte do Estado de Santa Catarina. 
relação ao fósforo orgânico, o que também ocorreu com a matéria orgânica total.

Os maiores percentuais de fosfato de ferro + alumínio (Fig. 8C) foram registrados próximos aos sistemas fluviais, enquanto que nestas mesmas áreas, o fosfato de cálcio (Fig. 8D) apresentou os menores índices. As maiores porcentagens de fosfato de cálcio estão relacionadas aos sedimentos marinhos mais distantes da linha de costa, e que, normalmente apresentam maior disponibilidade de material carbonático. Esta fração foi percentualmente predominante na maioria das amostras.

Comparando as distribuições percentuais de fosfato de ferro + alumínio (Fig. $8 \mathrm{C}$ ) e fosfato de cálcio (Fig. 8D) nota-se um gradiente bem definido com relação a profun- didade, evidenciando a contribuição continental do primeiro e, marinha do segundo.

De fato, os resultados mostram que a distribuição das formas de fósforo não pode ser explicada somente pela sua relação com o granulometria do sedimento. Também devem ser levadas em consideração as condições de deposição deste constituinte, as quais dependem de mecanismos de sedimentação e acumulação, que podem variar de acordo com as características biogeoquímicas da área de estudo. Assim sendo, um melhor detalhamento da distribuição de fósforo e suas formas neste local poderá ser obtido pela análise das amostras que foram excluídas na primeira seleção.
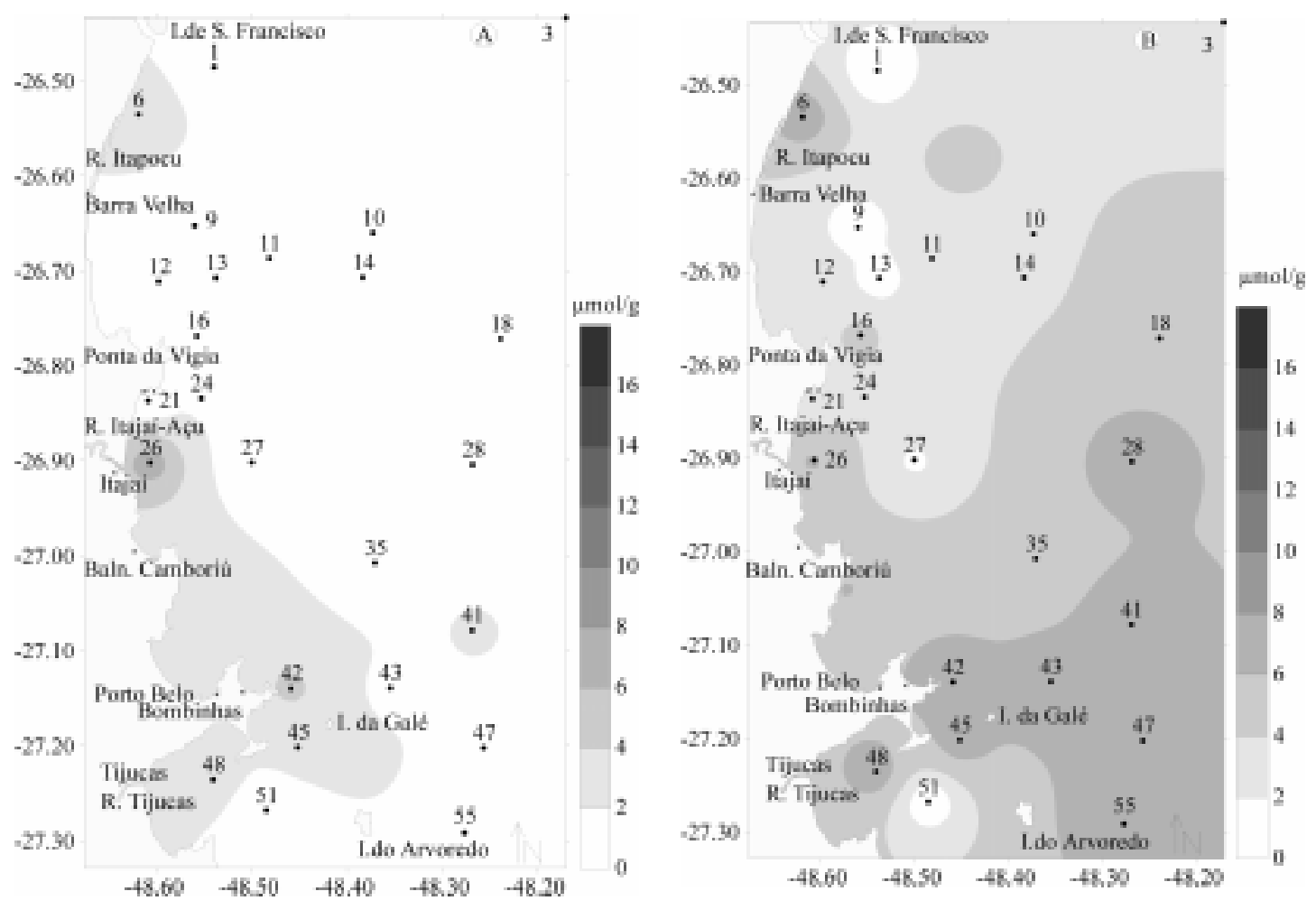

Figura 7 - Distribuição espacial de fosfato de ferro + alumínio (A) e fosfato de cálcio (B) (mmol/g), nos sedimentos superficiais da plataforma interna do Estado de Santa Catarina. 

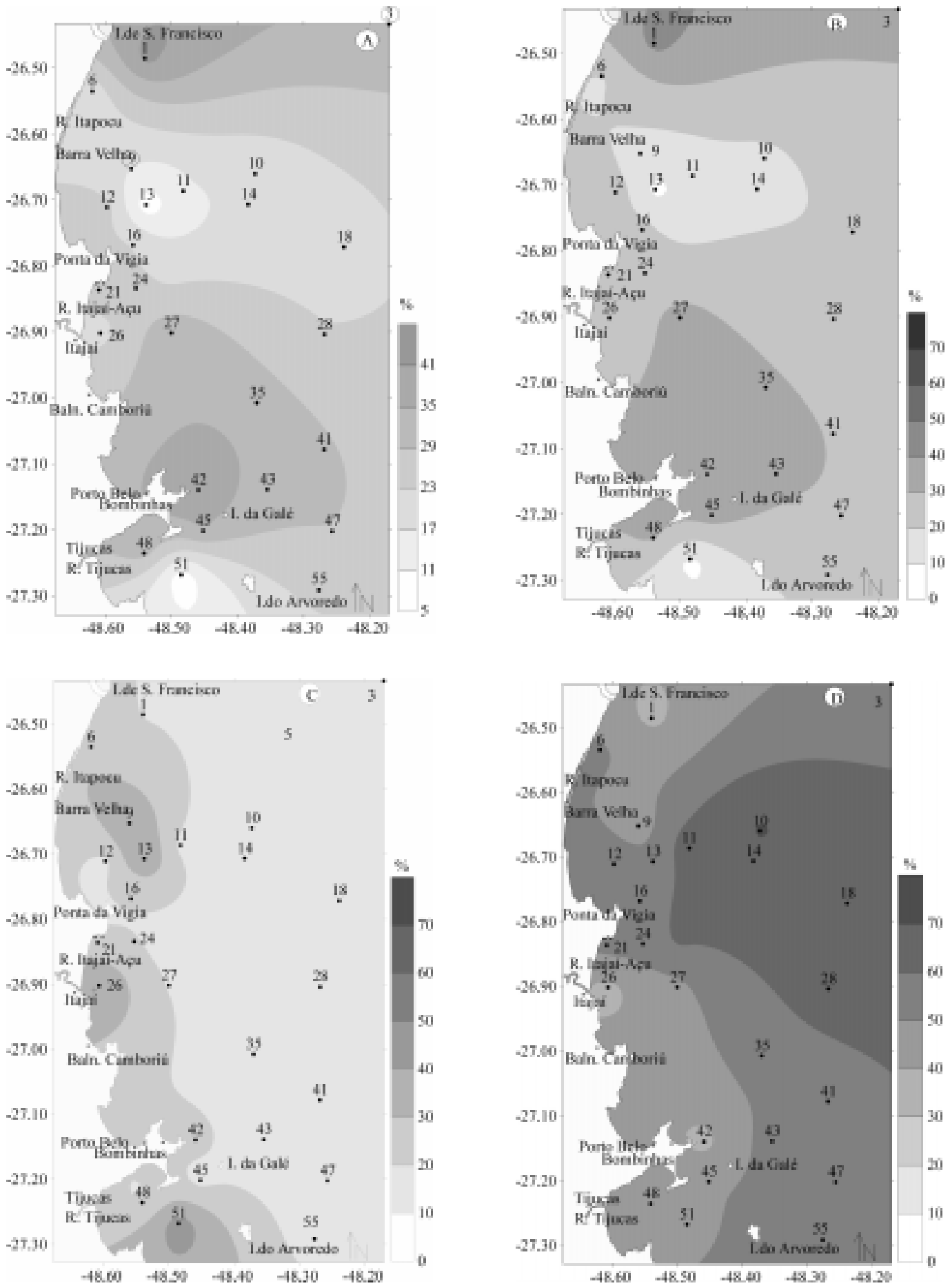

Figura 8 - Distribuição percentual de fósforo orgânico (A), inorgânico (B), fosfatos de ferro+alumínio $(C)$ e fosfato de cálcio (D), no sedimento superficial da plataforma interna do centro-norte de Santa Catarina. 


\section{CONCLUSÕES}

Na plataforma interna do litoral centronorte de Santa Catarina as concentrações de fósforo e suas formas foram maiores nos sedimentos finos que apresentam condições favoráveis a adsorção de fósforo.

As maiores concentrações de fosfato de ferro + alumínio ocorreram próximo a região costeira diminuindo com a profundidade, evidenciando aporte continental desta fração, que é derivada principalmente de erosão continental.

A forma inorgânica predominante foi o fosfato de cálcio que apresentou distribuição horizontal inversa a do ferro + alumínio, devido a sua origem marinha.

\section{AGRADECIMENTOS}

Os autores agradecem ao CEPSUL/ IBAMA (SC), ao Oc. Frederico César Leal Rodrigues pelos mergulhos realizados durante o embarque e, ao Conselho Nacional de Desenvolvimento Científico e Tecnológico (CNPq) pelo fornecimento de bolsa de iniciação científica.

\section{BIBLIOGRAFIA}

Barron, E.F. \& Frakes, L. 1990. Climate model evidence for variable significance for phosforite formation. In: Cambridge University Press, Phosphate Deposits of the World., 3: 260-272.

Bellotto, V.R.; Kuroshima, K.N; Rörig, L.R.1997. Projeto Integrado da Foz do Rio Itajaí-Açu e Área Costeira Adjacente: Diagnóstico e Avaliação Ambiental. v. II.

Berner, R. A. Diagenisis of $P$ in sediments from non-upwelling areas. 1990. In: Cambridge University Press, Phosphate Deposits of the World. 3: 27/32.

Bostrom, B; Andersen, J. M.; Fleischer, S. \& Jansson, M. 1988. Exchange of phosphorus across the sediment-water interface. Hidrobiologia. 180:229-244.

Chester, Roy. 1991. Marine Geochemistry. Cambridge University Press.

Dolan, J.R. Phosphorus and ammonia excretion by planktonic protists. 1997. Marine Geology. 139:109-122.

Logan, T. J. 1982. Mechanisms for release of sediment-bound phosphate to water and the effects of agricultural land management on fluvial transport of particulate and dissolved phosphate. Hidrobiologia. 92:519-530.

Odum, E. P. 1983. Ecologia. Editora Guanabara.

Peterson, K.; Bostron, B. \& Jacobsen, O. 1988. Phosphorus in sediments - speciation and analysis. Hidrobiologia. 170: 91-101.

Rao, L.J. \& Berner, R.A. 1997. Time variations of phosphorus and sources of sediments beneath the Chang Jiang (Yangtze river). Marine Geology. 139:95-108.

Silveira, I.M.O. 1993. Distribuição de fosfato e suas formas na plataforma continental. Dissertação de Mestrado. Universidade Federal Fluminense.

Strickland, J.D.H. \& Parsons, T.R. 1972. A practical handbook of seawater analysis. 2nd. J. Fish. Res. Board of Canada- Ottawa Bul.

Vink, S., Chambers, R.M. e Smith, S.V. Distribuition of phosphorus in sediments of Tomales Bay, Califórnia. 1997. Marine Geology. 139:157-179.

Williams, J.D.H.; Syers, J.K.; Shukla, S.S.; Harris, R.F. \& Armstrong, D.E. Levels of inorganic and total phosphurus in lake sediments related to othern sediment parameters. 1971. Enviromental Science \& Tecnology. 5:1113-1120. 